Journal of Statistical Physics manuscript No.

(will be inserted by the editor)

Paweł Góra · Zhenyang Li · Abraham

Boyarsky - Harald Proppe

\title{
Harmonic Averages and New Explicit Constants for Invariant Densities of Piecewise Expanding Maps of the Interval
}

Received: date / Accepted: date

\begin{abstract}
The statistical behavior of families of maps is important in studying the stability properties of chaotic maps. For a piecewise expanding map $\tau$ whose slope $>2$ in magnitude, much is known about the stability of the associated invariant density. However, when the map has slope $\leq 2$ many different behaviors can occur as shown in [?] for $\mathrm{W}$ maps. The main results of this note use a harmonic average of slopes condition to obtain new explicit constants for the upper and lower bounds of the invariant probability density function associated with the map, as well as a bound for the speed of convergence to the density. Since these constants are determined explicitly the results can be extended to families of approximating maps.
\end{abstract}

Keywords absolutely continuous invariant measures · piecewise expanding maps of interval · lower bound for invariant density - explicit constants for rate of convergence $\cdot$ harmonic average of slopes

Mathematics Subject Classification (2000) MSC 37A05 • MSC 37A10 • $37 \mathrm{E} 05$

The research of the authors was supported by NSERC grants.

Paweł Góra

Department of Mathematics and Statistics, Concordia University,

1455 de Maisonneuve Blvd. West, Montreal, Quebec H3G 1M8, Canada

E-mail: pgora@mathstat.concordia.ca

Zhenyang Li

E-mail: zhenyangemail@gmail.com

Abraham Boyarsky

E-mail: boyar@alcor.concordia.ca

Harald Proppe

E-mail: proppe@alcor.concordia.ca 


\section{Introduction}

Let $I=[0,1]$ and let $\mathcal{P}$ be a finite partition of $I$. Let $\mathcal{T}(I)$ denote the class of piecewise expanding transformations on $I$ with partition $\mathcal{P}$. We study statistical properties of the invariant probability density function (pdf) associated with $\tau$ in $\mathcal{T}(I)$. We impose two conditions on $\tau: 1$ ) weak covering, which means there exists an integer $K$ such that the union of forward images of every element of $\mathcal{P}$ equals $I$, and 2) harmonic average of slopes condition, which means that the harmonic average of the (inf of) slopes of every two adjoint intervals (except for the first and last interval) is strictly larger than 2. We use these two conditions to derive explicit constants for the upper and lower bounds of the invariant pdf as well as the constant that determines the speed of convergence to the invariant pdf. Related results were obtained in [?], but with the assumption that the magnitude of all slopes are strictly greater than 2. Without this condition many different behaviors for approximating maps can occur as shown in [?] for $\mathrm{W}$ maps. For example, the acims of approximating maps can converge to a singular, absolutely continuous or a mixed measure. By W map we mean any map with a graph in the shape of letter $\mathrm{W}$ for which the middle vertex is a fixed point. More precisely it is a map $\tau:[0,1] \rightarrow[0,1]$, piecewise monotonic on the partition $\left\{I_{1}, I_{2}, I_{3}, I_{4}\right\}$ of $[0,1], I_{i}=\left[a_{i_{1}}, a_{i}\right], i=1,2,3,4$, such that $\tau\left(a_{0}\right)=\tau\left(a_{4}\right)=1, \tau\left(a_{1}\right)=$ $\tau\left(a_{3}\right)=0$ and $\tau\left(a_{2}\right)=a_{2}$. An example of a $W$ map is shown in Figure ??. $\mathrm{W}$ maps are continuous but our considerations do not depend on continuity and we do not assume it.

It is one of the objectives of this paper to show that we can weaken the slope 2 condition using the harmonic average slope condition and establish stability of acim for some $\mathrm{W}$ maps.

In Section 2 we use the weak covering property and the harmonic average of slopes condition to derive an explicit bound on the number of iterations needed to obtain weak covering for any subinterval of a partition element. In Section 3 we use this result and a generalized Lasota-Yorke inequality to obtain explicit constants for the upper bound of the invariant pdf and from this we derive an explicit lower bound for the invariant pdf. We then show (Theorem ??) that we can extend our results to families of maps. We provide an example to show that the harmonic average of slopes condition is essential. For a W-shaped map example we calculate all the constants necessary to find the lower bound. In Section 4 we assume weak mixing and use our derived constants to find an explicit constant for the rate of convergence. Finding the rate of convergence of initial densities to the invariant pdf of the map is an important problem in many scientific fields. Our method depends on using equipartitions rather than partitions of the inverse images of $\mathcal{P}$ and, as such, in most situations, results in sharper constants. We work out an example where the results of [?] do not apply.

\section{Notation and Preliminary Results}

Let $I=[0,1]$ and let $m$ be Lebesgue measure on $I$. We present the usual definition of a piecewise expanding map. 
Definition 1 Suppose there exists a partition $\mathcal{P}=\left\{I_{i}:=\left[a_{i-1}, a_{i}\right], i=\right.$ $1, \ldots, q\}$ of $I$ such that $\tau: I \rightarrow I$ satisfies the following conditions:

1. $\tau_{i}:=\left.\tau\right|_{I_{i}}$ is $C^{1}$ and $\lim _{x \rightarrow a_{i-1}^{+}} \tau^{\prime}(x), \lim _{x \rightarrow a_{i}^{-}} \tau^{\prime}(x)$ exist (can be infinite);

2. $\left|\tau_{i}^{\prime}(x)\right| \geq s_{i}>1$ for any $i$ and for all $x \in\left(a_{i-1}, a_{i}\right)$.

If $\tau$ satisfies conditions $1-2$, we say it is in $\mathcal{T}(I)$, the class of piecewise expanding transformations.

We will also assume that $\tau$ is weakly covering, i.e.,

Definition 2 The map $\tau \in \mathcal{T}(I)$ is called weakly covering if and only if there exists a $K \geq 1$ such that

$$
\bigcup_{n=0}^{K} \tau^{n}\left(I_{i}\right)=[0,1], i=1, \ldots, q
$$

Let

$$
s:=\min _{1 \leq i \leq q} s_{i}>1
$$

Suppose $\tau \in \mathcal{T}(I)$ satisfies the following condition.

$$
s_{H}=\max _{i=1, \ldots, q-1}\left\{\frac{1}{s_{i}}+\frac{1}{s_{i+1}}\right\}<1 .
$$

The number $H(a, b)=\frac{2}{\frac{1}{a}+\frac{1}{b}}$ is called the harmonic average of $a$ and $b$. Condition $H(a, b)>2$ is equivalent to $\frac{1}{a}+\frac{1}{b}<1$. If $\tau$ satisfies $s_{H}<1$ we say that $\tau$ satisfies the harmonic average of slopes condition.

Now, we prove a very simple minimax lemma with important consequences.

Lemma 1 Let $z_{1}, z_{2}>1$ and $\alpha+\beta=c$, where $\alpha, \beta>0$. Assume

$$
\frac{1}{z_{1}}+\frac{1}{z_{2}}<1
$$

Then,

$$
\min _{\alpha, \beta} \max \left\{z_{1} \alpha, z_{2} \beta\right\}=\frac{1}{\frac{1}{z_{1}}+\frac{1}{z_{2}}} c>c .
$$

Proof We have

$$
\min _{\alpha, \beta} \max \left\{z_{1} \alpha, z_{2} \beta\right\}=\min _{\alpha} \max \left\{z_{1} \alpha, z_{2}(c-\alpha)\right\} .
$$

The line $f(\alpha)=z_{1} \alpha$ is increasing while the line $g(\alpha)=z_{2}(c-\alpha)$ is decreasing. The $\min _{\alpha} \max \left\{z_{1} \alpha, z_{2}(c-\alpha)\right\}$ occurs where the lines intersect, i.e., at

$$
\alpha=\frac{z_{2} c}{z_{1}+z_{2}}
$$

which gives

$$
\min _{\alpha, \beta} \max \left\{z_{1} \alpha, z_{2} \beta\right\}=\frac{z_{1} z_{2} c}{z_{1}+z_{2}}=\frac{1}{\frac{1}{z_{1}}+\frac{1}{z_{2}}} c>c .
$$


Remark 1 If $\frac{1}{z_{1}}+\frac{1}{z_{2}}=1$, then, $\min _{\alpha, \beta} \max \left\{z_{1} \alpha, z_{2} \beta\right\}=c$.

Lemma ?? implies

Proposition 1 If $\tau \in \mathcal{T}(I)$ satisfies the harmonic average of slopes condition, then for any subinterval $J \subset I$ which does not contain two endpoints of partition $\mathcal{P}$ we have

$$
m(\tau(J)) \geq \frac{1}{s_{H}} m(J) .
$$

Proof Note that

$$
s=\min _{1 \leq i \leq q} s_{i} \geq \min _{1 \leq i \leq q-1} \frac{1}{\frac{1}{s_{i}}+\frac{1}{s_{i+1}}} \geq \frac{1}{s_{H}} .
$$

If $J$ does not contain any endpoints of partition $\mathcal{P}$, then $J \subset I_{i}$, for some $1 \leq i \leq q$, and

$$
m(\tau(J)) \geq s_{i} m(J) \geq \frac{1}{s_{H}} m(J) .
$$

If $J$ contains exactly one endpoint of partition $\mathcal{P}$, then let $m(J)=\alpha+\beta$, where $\alpha$ and $\beta$ are the lengths of parts of $J$ to the left and to the right of the partition point, respectively. By Lemma ?? we obtain $m(\tau(J)) \geq \frac{1}{s_{H}} m(J)$.

Proposition 2 If $\tau \in \mathcal{T}(I)$ satisfies the harmonic average of slopes condition, then for any subinterval $J \subset I$ there exists a positive integer $M(J)$ such that at least one connected component of $\tau^{M(J)}(J)$ contains two endpoints of partition $\mathcal{P}$ and, automatically, the interval between them. Moreover, $M(J)$ satisfies

$$
0 \leq M(J) \leq \max \left\{\left\lceil\frac{\ln \frac{m(J)}{\delta_{\max }}}{\ln \left(s_{H}\right)}\right\rceil, 0\right\},
$$

where $\delta_{\max }=\max \left\{m\left(I_{i} \cup I_{i+1}\right) \mid i=1,2, \ldots, q-1\right\}$ and $\lceil t\rceil$ is the smallest integer equal or larger than $t$.

Proof Let $J$ be a subinterval of $I$. Then,

Case (i): If $J$ contains two or more endpoints of $\mathcal{P}$, then $M(J)=0$. In particular, this happens when $m(J) \geq \delta_{\max }$.

Case (ii): We assume $m(J)<\delta_{\max }$ and that $J$ contains at most one endpoint of partition $\mathcal{P}$. Let us assume that $J$ contains exactly one endpoint of $\mathcal{P}$, and this endpoint divides $J$ into two subintervals, $J_{0,1}$ and $J_{0,2}$. Lemma ?? implies

$$
\max \left\{m\left(\tau\left(J_{0,1}\right)\right), m\left(\tau\left(J_{0,2}\right)\right)\right\} \geq \frac{1}{s_{H}} m(J) .
$$

We can assume $m\left(\tau\left(J_{0,1}\right)\right) \geq \frac{1}{s_{H}} m(J)$. Notice that $\tau\left(J_{0,1}\right)$ is also an interval since $\tau \in \mathcal{T}(I)$.

If $J$ contains no endpoint of $\mathcal{P}$, then $\tau(J)$ is again an interval, and $m(\tau(J)) \geq \operatorname{sm}(J) \geq \frac{1}{s_{H}} m(J)$. 
Thus, for an interval $J$ that contains at most one endpoint of $\mathcal{P}$, we can find an interval in $\tau(J)$, denoted by $J_{1}$, such that $m\left(J_{1}\right) \geq \frac{1}{s_{H}} m(J)$. If $J_{1}$ contains two endpoints of $\mathcal{P}$, we stop the iteration. Otherwise, considering $\tau\left(J_{1}\right)$, we again find an interval in $\tau\left(J_{1}\right)$, denoted by $J_{2}$, such that $m\left(J_{2}\right) \geq$ $\frac{1}{s_{H}} m\left(J_{1}\right) \geq \frac{1}{s_{H}^{2}} m(J)$. Repeating this procedure, we can find an integer $k$ such that $m\left(J_{k}\right) \geq \frac{1}{s_{H}^{k}} m(J) \geq \delta_{\max }$, which implies that $\tau^{k}(J)$ contains at least two endpoints of $\mathcal{P}$. Therefore, we obtain

$$
M(J) \geq \frac{\ln \frac{m(J)}{\delta_{\max }}}{\ln \left(s_{H}\right)} .
$$

Corollary 1 If $\tau \in \mathcal{T}(I)$ is weakly covering and satisfies the harmonic average of slopes condition, then for any subinterval $J \subset I$ we have

$$
\bigcup_{n=0}^{K} \tau^{M(J)+n}(J)=[0,1]
$$

where $M(J)$ is defined in Proposition ??.

Remark 2 Note that the weak covering property plus $s_{H}<1$ does not imply topological exactness. The simplest example would be the map $\tau$ such that $\tau([0,1 / 2])=[1 / 2,1]$ and $\tau([1 / 2,1])=[0,1 / 2]$ and $\tau$ restricted to each of these intervals is a tent map. An additional assumption is needed for topological exactness. See Theorem ?? and Corollary ??.

We define $\mathcal{P}^{(n)}=\left\{I_{i_{0}} \cap \tau^{-1}\left(I_{i_{1}}\right) \cap \tau^{-2}\left(I_{i_{2}}\right) \cap \cdots \cap \tau^{-(n-1)}\left(I_{i_{n-1}}\right): 1 \leq\right.$ $\left.i_{0}, i-1, i-2, \ldots, i_{n-1} \leq q\right\}$. Partition $\mathcal{P}^{(n)}$ is the partition of monotonicity of $\tau^{n}$. Note that $\mathcal{P}=\mathcal{P}^{(1)}$.

For any $g:[0,1] \rightarrow \mathbb{R}$ we define its variation

$$
\bigvee_{[0,1]} g=\sup _{K} \sup \sum_{k=1}^{K}\left|g\left(s_{k-1}\right)-g\left(s_{k}\right)\right|,
$$

where the supremum is taken over all sequences $0=s_{0}<s_{1}<\cdots<s_{K}=1$. For more information about this notion, functions of bounded variation and their uses in the theory of piecewise expending maps of an interval, we refer the reader to [?].

Theorem 1 Let $\tau \in \mathcal{T}(I)$ be piecewise $C^{1+1}$ (see the definition at the beginning of the next section) with $s_{H}<1$ and assume inf $\phi \geq \beta>0$, where $\phi$ is the $\tau$-invariant density. If $\tau$ is weakly mixing (with respect to Lebesgue measure), then there exists $K_{1}$ such that

$$
\tau^{K_{1}}\left(I_{i}\right)=[0,1], i=1,2, \ldots, q .
$$


Proof We follow the proof of a similar theorem in [?]. For the maps we consider weak mixing is equivalent to mixing and to exactness [?] (all with respect to Lebesgue measure). Let $\chi=\chi_{I_{i}} / m\left(I_{i}\right)$ for some $1 \leq i \leq q$. Since $\tau$ is exact we have $P_{\tau}^{n} \chi \rightarrow \phi$ in $L^{1}$, as $n \rightarrow \infty$, where $P_{\tau}$ is the PerronFrobenius operator induced by $\tau$ [?]. Thus, for any $n_{1}$ (which will be fixed later) we can find an $N\left(n_{1}\right)$ such that for any $n \geq N\left(n_{1}\right)$ in every interval $J$ of the partition $\mathcal{P}^{\left(n_{1}\right)}$ there is a point $x \in J$ with $P_{\tau}^{n} \chi(x) \geq \beta / 2$. On the other hand, the Lasota-Yorke inequality implies that

$$
\bigvee_{[0,1]} P_{\tau}^{k} \chi \leq C
$$

for all $k$ and some constant $C$. Let $n \geq N\left(n_{1}\right)$ and

$$
\mathcal{B}=\left\{J \in \mathcal{P}^{\left(n_{1}\right)}: \exists_{x \in J} \text { such that } P_{\tau}^{n} \chi(x)<\beta / 4\right\} .
$$

If $J \in \mathcal{B}$, then we have $\bigvee_{J} P_{\tau}^{n} \chi \geq \beta / 4$ and

$$
\bigvee_{[0,1]} P_{\tau}^{n} \chi \geq(\beta / 4) \# \mathcal{B}
$$

Thus, $\# \mathcal{B} \leq 4 C / \beta=L_{0}$.

The Perron-Frobenius operator $P_{\tau}$ induced by $\tau$, can be viewed as an operator on $B V(I)$, the space of functions of bounded variation on $I$ (or more generally on $L^{1}(I)$ ). For $\tau \in \mathcal{T}(I)$ it has the following representation $[?]$

$$
P_{\tau} f=\sum_{i=1}^{q} \frac{f\left(\tau_{i}^{-1}(x)\right)}{\left|\tau^{\prime}\left(\tau_{i}^{-1}(x)\right)\right|} \chi_{\tau\left[a_{i-1}, a_{i}\right]}(x) .
$$

For more detailed information about the space $B V(I)$, operator $P_{\tau}$ and its properties we refer the reader to [?]. An important property of $P_{\tau}$ is that $f$ is an invariant pdf (or a $\tau$-invariant density) if and only if $P_{\tau} f=f$.

Using the representation of $P_{\tau}$, we have the following inequality for all $x \in[0,1] ;$

$$
\beta \leq \phi(x)=\sum_{y \in \tau^{-n}(x)} \frac{\phi(y)}{\left|\left(\tau^{n}\right)^{\prime}(y)\right|} \leq \sup (\phi) \#\left(\tau^{-n}(x)\right) s^{-n} .
$$

This shows that $\#\left(\tau^{-n}(x)\right)$ goes to infinity as $n$ goes to infinity, uniformly in $x$. In particular we can find an $N_{1}$ such that for all $x \in[0,1]$

$$
\#\left(\tau^{-N_{1}}(x)\right)>L_{0}
$$

Let us fix $n_{1}=N_{1}$ and $N_{2} \geq N\left(N_{1}\right)$. Then,

$$
P_{\tau}^{N_{1}+N_{2}} \chi(x)=\sum_{y \in \tau^{-N_{1}}(x)} \frac{P_{\tau}^{N_{2}} \chi(y)}{\left|\left(\tau^{N_{1}}\right)^{\prime}(y)\right|} \geq \frac{\beta}{4 s^{N_{1}}},
$$

since at least one preimage $y \in \tau^{-N_{1}}(x)$ belongs to an interval $J \notin \mathcal{B}$. 
We have proved that $\tau^{N_{1}+N_{2}}\left(I_{i}\right)=[0,1]$. Choosing $K_{1}$ to be the maximum of constants $N_{1}+N_{2}$ over all $i=1,2, \ldots, q$ completes the proof.

The following result is an immediate consequence.

Corollary 2 If $\tau \in \mathcal{T}(I)$ is weakly covering, weakly mixing and satisfies the harmonic average of slopes condition, then $\tau$ is topologically exact. For any subinterval $J \subset I$ we have

$$
\tau^{M(J)+K_{1}}(J)=[0,1],
$$

where $M(J)$ is the number from Proposition ?? and $K_{1}$ is the constant from Theorem ??.

\section{Lower bound for the invariant density}

From now on we assume that our $\tau \in \mathcal{T}(I)$ is piecewise $C^{1+1}$, i.e., each $\tau_{i}^{\prime}$ satisfies Lipschitz condition with a constant $M_{i}$ :

$$
\left|\tau_{i}^{\prime}(x)-\tau_{i}^{\prime}(y)\right| \leq M_{i}|x-y|, \text { for all } x, y \in I_{i}, i=1,2, \ldots, q .
$$

This means $\tau$ is a piecewise expanding, piecewise $C^{1+1}$ map of $I$. We introduce the following notation

$$
M:=\max _{1 \leq i \leq q} M_{i}
$$

and

$$
\delta_{i}^{ \pm}:=\delta_{\left\{\tau\left(a_{i}^{ \pm}\right) \notin\{0,1\}\right\}}= \begin{cases}0 & \text { if } \tau\left(a_{i}^{ \pm}\right) \in\{0,1\}, \\ 1 & \text { if } \tau\left(a_{i}^{ \pm}\right) \notin\{0,1\},\end{cases}
$$

where $\tau\left(a_{i}^{ \pm}\right)$means $\lim _{x \rightarrow a_{i}^{ \pm}} \tau\left(a_{i}\right)$. For example, $\delta_{i}^{+}=1$ means that the left endpoint of the $(i+1)$-st branch of $\tau$ is hanging (does not touch 0 or 1 ).

Also, let

$$
\eta_{i}:= \begin{cases}\max \left\{\frac{\delta_{0}^{+}}{s_{1}}, \frac{\delta_{1}^{+}}{s_{2}}\right\} & \text { if } i=1, \\ \max \left\{\frac{\delta_{q-1}^{-}}{s_{q-1}}, \frac{\delta_{q}^{-}}{s_{q}}\right\} & \text { if } i=q, \\ \max \left\{\frac{\delta_{i-1}^{-}}{s_{i-1}}, \frac{\delta_{i}^{+}}{s_{i+1}}\right\} & \text { for } i=2 \ldots q-1 .\end{cases}
$$

Now, we present the following stronger version of the Lasota-Yorke inequality [?].

Proposition 3 Let $\tau \in \mathcal{T}(I)$, and satisfy the Lipschitz condition (??). Then, for every $f \in B V([0,1])$,

$$
\bigvee_{I} P_{\tau} f \leq \eta \bigvee_{I} f+\gamma \int_{I}|f| d m
$$

where $\eta=\max _{1 \leq i \leq q}\left\{\frac{1}{s_{i}}+\eta_{i}\right\}, \gamma=\frac{M}{s^{2}}+\frac{2 \max _{1 \leq i \leq q} \eta_{i}}{\min _{1 \leq i \leq q} m\left(I_{i}\right)}$. 


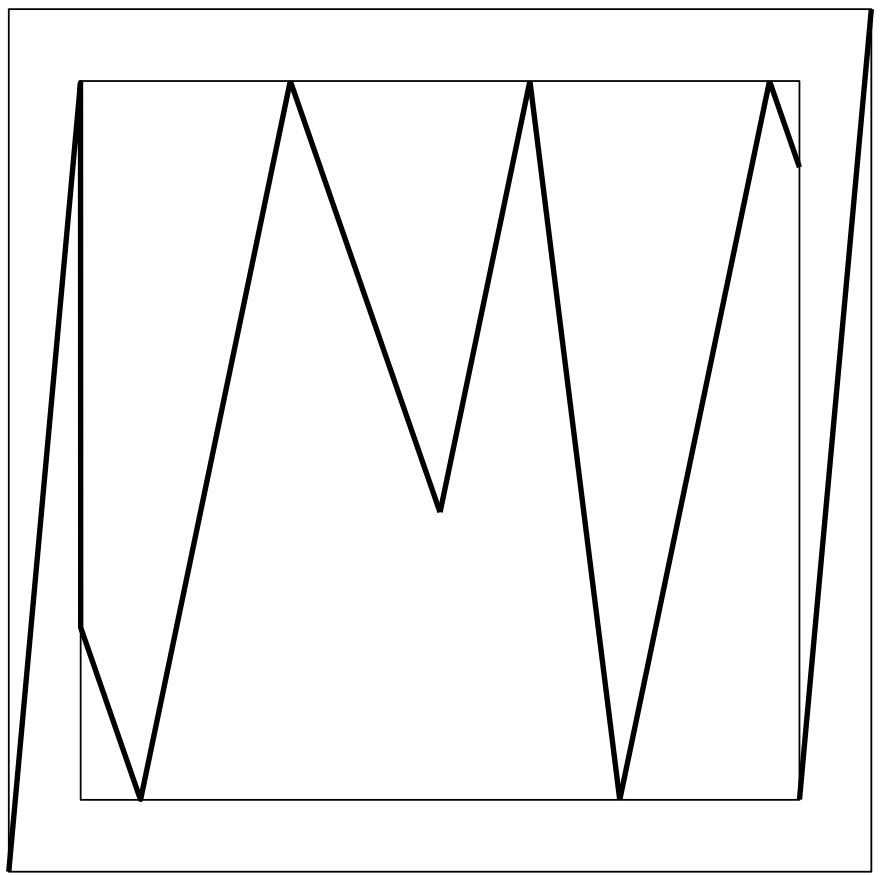

Fig. 1 The extension of map $\tau$ to $[0-\varepsilon, 1+\varepsilon]$.

Note that we always have $\max _{1 \leq i \leq q} \eta_{i}<\frac{1}{s}$.

As proved in Theorem 3.2 of [?], if $\tau(0), \tau(1) \in\{0,1\}$, then $\eta \leq s_{H}<1$. If the condition $\tau(0), \tau(1) \in\{0,1\}$ is not satisfied one uses an extension method to arrive at a similar conclusion, as done in Theorem 3.3 of [?]. For completeness, we describe the method. Let $I^{\varepsilon}=[0-\varepsilon, 1+\varepsilon]$ for some fixed small positive $\varepsilon$ and define $\tau^{\varepsilon}$ on $I^{\varepsilon}$ as follows

$$
\tau^{\varepsilon}(x)= \begin{cases}-\varepsilon+\frac{1+\varepsilon}{\varepsilon}(x+\varepsilon) & , x \in[-\varepsilon, 0) \\ \tau(x) & , x \in[0,1] \\ 0+\frac{1+\varepsilon}{\varepsilon}(x-1) & , x \in(1,1+\varepsilon]\end{cases}
$$

See Figure ?? for an illustration. The interval $[0,1]$ is the attractor of $\tau^{\varepsilon}$. We choose $\varepsilon$ so small that the constants $s$ and $s_{H}$ are the same for maps $\tau$ and $\tau^{\varepsilon}$. We consider the subspace $B V^{\varepsilon}\left(I^{\varepsilon}\right)=\left\{f \in B V\left(I^{\varepsilon}\right): f(x)=\right.$ 0 outside $[0,1]\}$ of $B V\left(I^{\varepsilon}\right)$. It is easy to check that $P_{\tau^{\varepsilon}}\left(B V^{\varepsilon}\left(I^{\varepsilon}\right)\right) \subset B V^{\varepsilon}\left(I^{\varepsilon}\right)$ and $\left(P_{\tau^{\varepsilon}} f\right)_{\mid[0,1]}=P_{\tau}\left(f_{\mid[0,1]}\right)$ for $f \in B V^{\varepsilon}\left(I^{\varepsilon}\right)$. Now, we obtain inequality (??) for $P_{\tau^{\varepsilon}}$ on $B V\left(I^{\varepsilon}\right)$. In particular it holds for $f \in B V^{\varepsilon}\left(I^{\varepsilon}\right)$. The constants $\eta_{i}$ are different but by the choice of $\varepsilon$ we still have $\eta<s_{H}$ and $\max _{1 \leq i \leq q} \eta_{i}<\frac{1}{s}$. The additional partition subintervals $I_{0}=[-\varepsilon, 0]$ and $I_{q+1}=[1,1+\varepsilon]$ do not appear in the $\min _{1 \leq i \leq q} m\left(I_{i}\right)$ because the integrals $\int_{I_{0}} f d m$ and $\int_{I_{q+1}} f d m$ are 0 
for $f \in B V^{\varepsilon}\left(I^{\varepsilon}\right)$. Thus, for $f \in B V^{\varepsilon}\left(I^{\varepsilon}\right)$, we obtain the inequality

$$
\bigvee_{I^{\varepsilon}} P_{\tau} f \leq \eta \bigvee_{I^{\varepsilon}} f+\gamma \int_{I}|f| d m
$$

with $\eta \leq s_{H}<1$ and $\gamma=\frac{M}{s^{2}}+\frac{2}{s \cdot \min _{1<i<q} m\left(I_{i}\right)}$.

It is well known (see [?]) that (??) or (??) implies that $\tau$ admits an acim with a pdf of bounded variation. We denote this invariant density by $\phi$. It follows from (??) or (??) that

$$
\bigvee_{I} \phi \leq \frac{\gamma}{1-\eta}
$$

We now consider the uniform partition $\mathcal{P}^{u}$ of $[0,1]$ into $2\left(\left[\frac{\gamma}{1-\eta}\right]+1\right)$ subintervals, where $\left[\frac{\gamma}{1-\eta}\right]$ is the integer part of $\frac{\gamma}{1-\eta}$. Thus, for each $J \in \mathcal{P}^{u}$, we have $m(J)<\frac{1-\eta}{2 \gamma}$. Now, we prove:

Lemma 2 There exists $J_{u} \in \mathcal{P}^{u}$ such that

$$
\phi(x) \geq \frac{1}{2} \quad \text { for all } x \in J_{u} .
$$

Proof Suppose the conclusion is not true. Then, for each $J \in \mathcal{P}^{u}$, there exists a point $x_{J} \in J$ such that $\phi\left(x_{J}\right)<\frac{1}{2}$. Using the inequality (??), we obtain

$$
\begin{aligned}
1 & =\int_{I} \phi d m=\sum_{J \in \mathcal{P}^{u}} \int_{J} \phi d m \leq \sum_{J \in \mathcal{P}^{u}} m(J)\left(\phi\left(x_{J}\right)+\bigvee_{J} \phi\right) \\
& <\sum_{J \in \mathcal{P}^{u}}\left(\frac{m(J)}{2}+\frac{1-\eta}{2 \gamma} \bigvee_{J} \phi\right)=\frac{1}{2}+\frac{1-\eta}{2 \gamma} \bigvee_{I} \phi \leq \frac{1}{2}+\frac{1-\eta}{2 \gamma} \frac{\gamma}{1-\eta}=1 .
\end{aligned}
$$

The contradiction completes the proof.

Now, we can prove the existence of the lower bound for the invariant pdf of $\tau$. This result for individual maps is not new, see [?], [?] or [?]. What is new are the explicit constants we obtain, which allows us to prove the existence of the uniform lower bound for the invariant densities of a family of maps.

Theorem 2 Let $\tau \in \mathcal{T}(I)$ be piecewise $C^{1+1}$ and satisfy $s_{H}<1$. Then there exists $\beta>0$ such that inf $\phi \geq \beta$, where $\phi$ is the $\tau$-invariant density.

Proof Let $S_{\max }$ denote the biggest value of $\left|\tau^{\prime}(x)\right|$ over I. Since $\phi$ is the invariant density, $P_{\tau}^{n} \phi=\phi$ for any natural number $n$. Lemma ?? implies that there exists interval $J_{u} \subseteq I$ with $m\left(J_{u}\right)=\frac{1}{\left.2\left(\frac{1}{1-\eta}\right]+1\right)}$ such that

$$
\phi(y) \geq \frac{1}{2} \quad \text { for all } y \in J_{u} .
$$


By Corollary (??), for each $x \in I$, we can find an integer $n_{u} \leq M\left(J_{u}\right)+K$ and $y_{u} \in J_{u}$ such that $\tau^{n_{u}}\left(y_{u}\right)=x$. Therefore,

$$
\phi(x)=\left(P_{\tau}^{n_{u}} \phi\right)(x)=\sum_{y \in \tau^{-n_{u}}(x)} \frac{\phi(y)}{\left|\left(\tau^{n_{u}}\right)^{\prime}(y)\right|} \geq \frac{\phi\left(y_{u}\right)}{\left|\left(\tau^{n_{u}}\right)^{\prime}\left(y_{u}\right)\right|} \geq \frac{1}{2 S_{\max }^{n_{u}}} .
$$

Setting $\beta=\left(2 S_{\max }^{n_{u}}\right)^{-1}$ (or $\beta=\left(2 S_{\max }^{M\left(J_{u}\right)+K}\right)^{-1}$ for an explicit formula) completes the proof.

The next theorem generalizes Theorem ?? to a family of maps uniformly satisfying the assumptions.

Theorem 3 Let $\left\{\tau^{(r)}\right\} \subset \mathcal{T}(I)$ be a family of piecewise $C^{1+1}$ maps. The defining partition for $\tau^{(r)}$ is $\mathcal{P}^{(r)}=\left\{I_{1}^{(r)}, \ldots, I_{q(r)}^{(r)}\right\}$. We assume we can find uniform constants $s_{H}<1, K, \delta>0, \delta_{\max }, M, s>1, S_{\max }$ such that

$$
\begin{aligned}
s_{H} & \geq s_{H}^{(r)}=\max \left\{\left(\min _{I_{i}^{(r)}} \mid\left(\tau^{(r)}\right)^{\prime-1}+\left(\min _{I_{i+1}^{(r)}} \mid\left(\tau^{(r)}\right)^{\prime-1}: i=1,2, \ldots, q(r)-1\right\}\right.\right. \\
K & \geq K^{(r)}, \text { where } \cup_{n=0}^{K^{(r)}}\left(\tau^{(r)}\right)^{n}\left(I_{i}^{(r)}\right)=[0,1], i=1,2, \ldots, q(r) ; \\
\delta & \leq \delta^{(r)}=\min \left\{m\left(I_{i}^{(r)}\right): i=1,2, \ldots, q(r)\right\} \\
\delta_{\max } & \geq \delta_{\max }^{(r)}=\max \left\{m\left(I_{i}^{(r)} \cup I_{i+1}^{(r)}\right): i=1,2, \ldots, q(r)-1\right\} \\
M & \geq M^{(r)}, \text { the common Lipschitz constant for }\left(\tau_{i}^{(r)}\right)^{\prime}, i=1,2, \ldots, q(r) \\
s & \leq s^{(r)}=\min \left\{\min _{I_{i}^{(r)}}\left|\left(\tau_{i}^{(r)}\right)^{\prime}\right|, i=1,2, \ldots, q(r)\right\} \\
S_{\max } & \geq S_{\max }^{(r)}=\max \left\{\max _{I_{i}^{(r)}}\left|\left(\tau_{i}^{(r)}\right)^{\prime}\right|, i=1,2, \ldots, q(r)\right\}
\end{aligned}
$$

Let us define

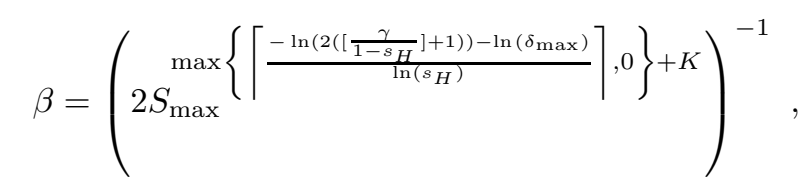

where $\gamma=\frac{M}{s^{2}}+\frac{2}{s \cdot \delta}$. Then, for all $r$, inf $\phi^{(r)} \geq \beta$, where $\phi^{(r)}$ is the $\tau^{(r)}$ invariant density.

Proof Combination of previous results in the paper. 
Below we refer to an example from [?] which shows that the condition $s_{H}<1$ is necessary in Theorem ??. Another such example was constructed in $[?]$.

Example 1 In [?] there was constructed a family $\left\{\tau^{(r)}\right\}$ of W-shaped maps converging to the standard $W$-map $\tau_{0}$ with a turning fixed point at $1 / 2$ and slopes 2 to the left of $1 / 2$ and -2 to the right of this point. The uniform constants $K, \delta>0, \delta_{\max }, M, s>1, S_{\max }$ can be found for this family. The constants $s_{H}^{(r)}$ converge to 1 , as $\tau^{(r)} \rightarrow \tau_{0}$. Each $\tau^{(r)}$ is exact on the whole $[0,1]$, but the absolutely continuous invariant measures of $\tau^{(r)}$ converge to Dirac measure $\delta_{(1 / 2)}$ as $\tau^{(r)} \rightarrow \tau_{0}$. Thus, the uniform positive lower bound cannot exist for the invariant densities of this family.

We now present an example of a non-linear W-shaped map and calculate for it all the constants necessary to find the lower bound. The theoretical bound is approximately $5.53 \times 10^{-14}$, while the computer simulation indicates that the lower bound is 0.54 .

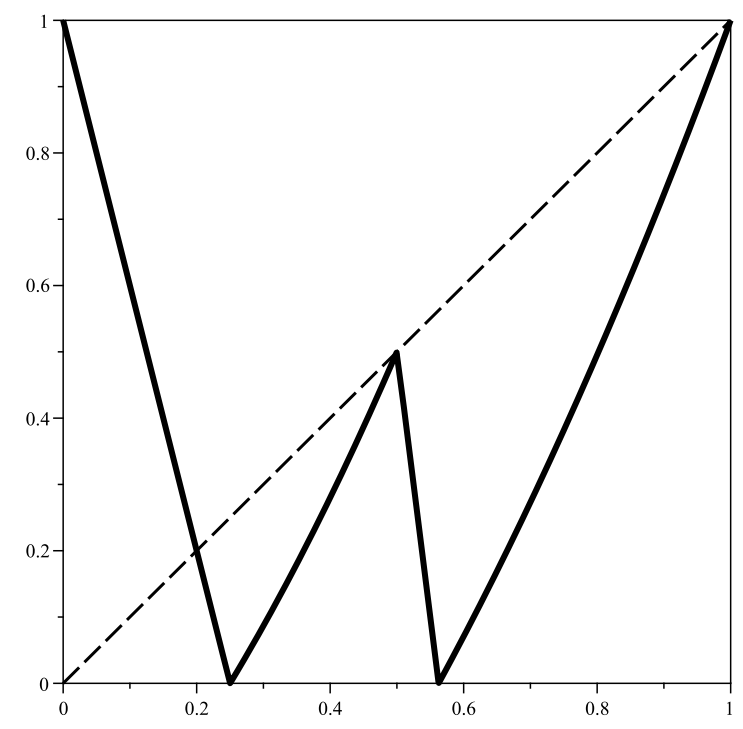

Fig. 2 Non piecewise linear W-shaped map.

Example 2 Let the map $\tau$ be defined as follows

$$
\tau(x)= \begin{cases}\tau_{1}(x):=1-40 / 9 x, & 0 \leq x<9 / 40 \\ \tau_{2}(x):=2(x-9 / 40), & 9 / 40 \leq x<9 / 20 \\ \tau_{3}(x):=-4(x-9 / 16), & 9 / 20 \leq x<9 / 16 \\ \tau_{4}(x):=x^{2}+81 / 112 x-81 / 112, & 9 / 16 \leq x<1 .\end{cases}
$$


The graph of $\tau$ is shown in Figure ??. We have

$$
\begin{aligned}
\tau_{1}^{\prime}(x) & =-40 / 9, \quad \tau_{2}^{\prime}(x)=2, \quad \tau_{3}^{\prime}(x)=-4, \quad \tau_{4}^{\prime}(x)=2 x+81 / 112 ; \\
s_{1} & =40 / 9, \quad s_{2}=2, \quad s_{3}=4, \quad s_{4}=207 / 112 ; \\
s & =\min \left\{40 / 9, s_{2}=2, s_{3}=4, s_{4}=207 / 112\right\}=207 / 112 ; \\
m\left(I_{1}\right) & =m([0,9 / 40))=9 / 40, \quad m\left(I_{2}\right)=m([9 / 40,9 / 20))=9 / 40, \\
m\left(I_{3}\right) & =m([9 / 20,9 / 16))=9 / 80, \quad m\left(I_{4}\right)=m([9 / 16,1])=7 / 16 ; \\
\delta & =\min \left\{m\left(I_{1}\right), m\left(I_{2}\right), m\left(I_{3}\right), m\left(I_{4}\right)\right\}=9 / 80 ; \\
\delta_{\max } & =\max \left\{m\left(I_{1}\right)+m\left(I_{2}\right), m\left(I_{2}\right)+m\left(I_{3}\right), m\left(I_{3}\right)+m\left(I_{4}\right)\right\} \\
& =\max \{9 / 20,27 / 80,11 / 20\}=11 / 20 ; \\
s_{H} & =\max \{9 / 40+1 / 2,1 / 2+1 / 4,1 / 4+112 / 207\}=655 / 828 ; \\
M_{1} & =0, \quad M_{2}=0, \quad M_{3}=0, \quad M_{4}=2 ; \\
M & =\max \{0,0,0,2\}=2 ; \\
\gamma & =\frac{M}{s^{2}}+\frac{2}{s \cdot \delta}=437248 / 42849 ; \\
{\left[\gamma /\left(1-s_{H}\right)\right] } & =[1748992 / 35811]=48 ; \\
S_{\max } & =40 / 9 ; \quad m\left(J_{u}\right)=\frac{1}{98} ; \quad K=2 .
\end{aligned}
$$

The estimate for number $N_{u}$ of iterations needed for any interval $J_{u}$ to expand to the whole $[0,1]$ comes from Corollary ?? and is

$$
\begin{aligned}
N_{u} \geq \max \left\{\left\lceil\frac{-\ln \left(2\left(\left[\frac{\gamma}{1-s_{H}}\right]+1\right)\right)-\ln \left(\delta_{\max }\right)}{\ln \left(s_{H}\right)}\right\rceil, 0\right\}+K \\
=\left[\frac{\ln (539 / 10)}{\ln (828 / 655)}\right]+1+2=20,
\end{aligned}
$$

which gives

$$
\beta \geq\left(2 S_{\max }^{N_{u}}\right)^{-1}=\left(2(40 / 9)^{20}\right)^{-1} \approx 5.53 \times 10^{-14} .
$$

With the aid of a computer we found the actual value $N_{u}=8$, which gives a much better, although still perhaps unsatisfactory estimate $\beta \geq 3.28 \times 10^{-6}$.

\section{Explicit convergence constants}

In this section we assume that $\tau \in \mathcal{T}(I)$ is weakly covering, weakly mixing, and piecewise of class $C^{1+1}$ with $s_{H}<1$. In particular, this implies Theorem ??, Corollary ?? and Theorem ??. To obtain the exact convergence constant, we follow the method of Liverani [?] with small improvements. For more information on Hilbert metrics and the use of cones in the theory of piecewise expanding maps we refer the reader to [?], [?] or [?]. 
We consider the following cone:

$C_{\kappa}=\left\{g(x) \in B V(I) \mid g(x) \neq 0, g(x) \geq 0\right.$ for all $\left.x \in[0,1] ; \bigvee_{[0,1]} g \leq \kappa \int_{[0,1]} g d m\right\}$

Let $\theta=\eta+\frac{\gamma}{\kappa}$.

Lemma 3 If $\kappa>\frac{\gamma}{1-\eta}$, then $\theta<1$ and $P_{\tau} C_{\kappa} \subset C_{\theta \kappa}$.

Proof First, $\theta=\eta+\frac{\gamma}{\kappa}<\eta+\gamma \frac{1-\eta}{\gamma}=1$.

If $f \in C_{\kappa}$, using (??), we obtain

$$
\bigvee_{[0,1]} P_{\tau} f \leq \eta \bigvee_{[0,1]} f+\gamma \int_{[0,1]}|f| d m \leq(\eta \kappa+\gamma) \int_{[0,1]}|f| d m=\kappa \theta \int_{[0,1]}|f| d m
$$

Lemma ?? shows that the cone $C_{\kappa}$ is invariant under the action of the operator $P_{\tau}$. We now define the Hilbert metric $\Theta(f, g)$ on $C_{\kappa}$. For $f, g$ in $C_{\kappa}$ let

$$
\begin{aligned}
& \alpha(f, g)=\sup \{\lambda>0 \mid \lambda f \leq g\} \\
& \beta(f, g)=\inf \{\mu>0 \mid g \leq \mu f\} \\
& \Theta(f, g)=\ln \left(\frac{\beta(f, g)}{\alpha(f, g)}\right)
\end{aligned}
$$

where we set $\alpha=0$ or $\beta=\infty$ when the corresponding sets are empty.

We recall the following lemma from [?].

Lemma 4 If $\Theta_{\kappa}$ is the Hilbert metric associated with the cone $C_{\kappa}$, then for each $\nu<1$ and $g \in C_{\kappa \nu}$

$$
\Theta_{\kappa}(g, 1) \leq \ln \left(\frac{\max \left\{(1+\nu) \int_{[0,1]} g d m, \sup _{x \in[0,1]} g(x)\right\}}{\min \left\{(1-\nu) \int_{[0,1]} g d m, \inf _{x \in[0,1]} g(x)\right\}}\right) .
$$

A slight change in Lemma ?? yields:

Lemma 5 Let $\mathcal{P}^{u}$ be the uniform partition of $[0,1]$ into $2\left(\left[\frac{\gamma}{1-\eta}\right]+1\right)$ subintervals. For each $g \in C_{\kappa}$, there exists $J_{u^{*}} \in \mathcal{P}^{u}$ such that

$$
g(x) \geq \frac{1}{2} \int_{[0,1]} g d m \quad \text { for all } x \in J_{u^{*}} .
$$


Proof Consider the normalized function, $\frac{g(x)}{\int_{[0,1]} g d m}$, which is a density function and also in $C_{\kappa}$. Lemma ?? implies that there exists $J_{u^{*}} \in \mathcal{P}^{u}$ such that

$$
\frac{g(x)}{\int_{[0,1]} g d m} \geq \frac{1}{2} \quad \text { for all } x \in J_{u^{*}}
$$

This completes the proof.

Let $M\left(J_{u^{*}}\right)$ and $K_{1}$ be as in Proposition ?? and Theorem ??. We now prove

Lemma 6 For each $\kappa>\frac{\gamma}{1-\eta}$, there exists $N_{u^{*}} \geq M\left(J_{u^{*}}\right)+K_{1}$ and $\Delta>0$ such that

$$
\operatorname{diam}\left(P_{\tau}^{N_{u^{*}}}\left(C_{\kappa}\right)\right) \leq \Delta<\infty .
$$

Proof Let $g(x) \in C_{\kappa}$, Lemma ?? implies that there exists $J_{u^{*}} \in \mathcal{P}^{u}$ such that $\frac{g(x)}{\int_{[0,1]} g d m} \geq \frac{1}{2}$ for all $x \in J_{u^{*}}$. Corollary ?? implies that we can find an integer $N_{u^{*}} \leq M\left(J_{u^{*}}\right)+K_{1}$ and $y_{u^{*}} \in J_{u^{*}}$ such that $\tau^{N_{u^{*}}}\left(y_{u^{*}}\right)=x$. Therefore,

$$
\left(P_{\tau}^{N_{u^{*}}} g\right)(x)=\sum_{y \in \tau^{-N_{u^{*}}(x)}} \frac{g(y)}{\left|\left(\tau^{N_{u^{*}}}\right)^{\prime}(y)\right|} \geq \frac{g\left(y_{u^{*}}\right)}{\left|\left(\tau^{N_{u^{*}}}\right)^{\prime}\left(y_{u^{*}}\right)\right|} \geq \frac{\int_{[0,1]} g d m}{2 S_{\max }^{N_{u^{*}}}} .
$$

Using Lemma ??, we obtain $P_{\tau}^{N_{u^{*}}} C_{\kappa} \subset C_{\theta_{1} \kappa}$, where

$$
\theta_{1}=\eta^{N_{u^{*}}}+\frac{1-\eta^{N_{u^{*}}}}{1-\eta} \frac{\gamma}{\kappa} .
$$

Let

$$
\omega(g)=\frac{\inf _{x \in[0,1]}\left(P_{\tau}^{N_{u^{*}}} g\right)(x)}{\int_{[0,1]} g d m} .
$$

Then,

$$
\frac{1}{2 S_{\max }^{N_{u^{*}}}} \leq \omega(g) \leq 1
$$

Note that

$$
\bigvee_{I} P_{\tau}^{N_{u^{*}}} g \leq \eta^{N_{u^{*}}} \bigvee_{I} g+\frac{1-\eta^{N_{u^{*}}}}{1-\eta} \gamma \int_{[0,1]} g d m
$$

which implies

$$
\frac{\bigvee_{I} P_{\tau}^{N_{u^{*}}} g}{\int_{[0,1]} g d m} \leq \kappa \theta_{1}
$$


Using Lemma ??, we obtain

$$
\begin{aligned}
& \operatorname{diam}\left(P_{\tau}^{N_{u^{*}}}\left(C_{\kappa}\right)\right) \\
& \leq \sup _{g \in P_{\tau}^{N_{u^{*}}}\left(C_{\kappa}\right)} 2 \ln \left(\frac{\max \left\{\left(1+\theta_{1}\right) \int_{[0,1]} P_{\tau}^{N_{u^{*}}} g d m, \sup _{x \in[0,1]}\left(P_{\tau}^{N_{u^{*}}} g\right)(x)\right\}}{\min \left\{\left(1-\theta_{1}\right) \int_{[0,1]} P_{\tau}^{N_{u^{*}}} g d m, \inf _{x \in[0,1]}\left(P_{\tau}^{N_{u^{*}}} g\right)(x)\right\}}\right) \\
& \leq \sup _{g \in P_{\tau}^{N_{u^{*}}}\left(C_{\kappa}\right)} 2 \ln \left(\frac{\max \left\{\left(1+\theta_{1}\right) \int_{[0,1]} g d m, \inf _{x \in[0,1]}\left(P_{\tau}^{N_{u^{*}}} g\right)(x)+\bigvee_{I} P_{\tau}^{N_{u^{*}} g}\right\}}{\min \left\{\left(1-\theta_{1}\right) \int_{[0,1]} g d m, \inf _{x \in[0,1]}\left(P_{\tau}^{N_{u^{*}}} g\right)(x)\right\}}\right) \\
& \leq 2 \ln \left(\frac{\max \left\{1+\theta_{1}, 1+\kappa \theta_{1}\right\}}{\min \left\{1-\theta_{1}, \frac{1}{2 S_{\max }^{N_{u}^{*}}}\right\}}\right) \equiv \Delta \text {. }
\end{aligned}
$$

Thus, exactly as in [?], we obtain the following theorem on the decay of correlations.

Theorem 4 Let $\tau \in \mathcal{T}(I)$ be weakly covering, weakly mixing and piecewise of class $C^{1+1}$ with $s_{H}<1$. Then, for each $f \in L^{1}([0,1])$ and a density $g \in B V([0,1])$,

$$
\left|\int_{[0,1]} g \cdot f \circ \tau^{n} d m-\int_{[0,1]} f d \mu\right| \leq K_{n} \Lambda^{n}|| f \|_{1}\left(1+b \bigvee_{[0,1]} g\right)
$$

where

$$
\begin{gathered}
\Lambda=\tanh (\Delta / 4)^{\frac{1}{N_{u^{*}}}}, \\
K_{n}=\exp \left(\Delta \Lambda^{n-N_{u^{*}}}\right) \Lambda^{-N_{u^{*}}} \Delta\|\phi\|_{\infty}, \\
b=\left(\kappa-\frac{\gamma}{1-\eta}\right)^{-1}
\end{gathered}
$$

Note that $|\phi| \leq \bigvee_{[0,1]} \phi+\frac{\|\phi\|_{1}}{1-0} \leq \gamma /(1-\eta)+1$ and since $\Lambda<1$, we have $\lim _{n \rightarrow \infty} K_{n} \leq \Lambda^{-N_{u^{*}}} \Delta(\kappa+1)$. Although we may not have an explicit formula for $N_{u^{*}}$, we can give a sufficient lower bound using Proposition ??

In [?] the convergence constants are calculated for an example. We calculated them for the same example and obtained the same numbers. For maps with constant modulus of slope and without turning periodic points our method does not offer any advantages over the methods of [?] or [?]. Below, we continue Example ?? to which the methods of [?] and [?] do not apply. 
Example ??. (continued) We use the directly calculated $N_{u^{*}}=8$. We have $\gamma /(1-\eta)=\gamma /\left(1-s_{H}\right)=1748992 / 35811$. We choose $\kappa=1748995 / 35811$. By equation (??), we have $\theta_{1} \sim 0.9999985478$ and

$$
\Delta=2 \ln \left(\left(1+\kappa \theta_{1}\right) 2 S_{\max }^{N_{u^{*}}}\right) \sim 33.07038934 .
$$

Then, $\Lambda \sim 0.9999999835, b=11937$ and

$$
K_{n} \leq \sim 1648 \exp \left(33 \cdot 0.9999999835^{n-8}\right) .
$$

Since all the constants in Theorem ?? are explicit we obtain a similar theorem for families.

Theorem 5 Let a family $\left\{\tau^{(r)}\right\}$ satisfy the assumptions of Theorem ??. We assume that all maps $\tau^{(r)}$ are weakly mixing with uniform constant $K_{1}$ of Theorem ??. Then, Theorem ?? holds for family $\left\{\tau^{(r)}\right\}$ with uniform constants $\Lambda, b$ and $K_{n}$.

Acknowledgment: The authors are grateful to anonymous reviewers for detailed comments that improved the presentation of the paper.

\section{References}

1. Baladi, V., Positive transfer operators and decay of correlations, Advanced Series in Nonlinear Dynamics, 16. World Scientific Publishing Co., Inc., River Edge, NJ, 2000. x+314 pp. ISBN: 981-02-3328-0.

2. Boyarsky, A. and Góra, P., Laws of Chaos. Invariant Measures and Dynamical Systems in One Dimension, Probability and its Applications, Birkhäuser, Boston, MA, 1997.

3. Eslami, P. and Góra, P., Stronger Lasota-Yorke inequality for piecewise monotonic transformations, preprint.

4. Eslami, P. and Misiurewicz, M., Singular limits of absolutely continuous invariant measures for families of transitive map, Journal of Difference Equations and Applications, (2011), doi:10.1080/10236198.2011.590480.

5. Keller, G., Stochastic stability in some chaotic dynamical systems, Monatshefte für Mathematik 94 (4) (1982) 313-333.

6. Keller, G., Piecewise monotonic transformations and exactness, Collection: Seminar on Probability, Rennes 1978 (French) Exp. No. 6, 32 pp., Univ. Rennes, Rennes.

7. Keller, G., Interval maps with strictly contracting Perron-Frobenius operators, Internat. J. Bifur. Chaos Appl. Sci. Engrg. 9 (1999), No. 9, 1777-1783.

8. Keller, G. and Liverani, C., Stability of the spectrum for transfer operators, Ann. Scuola Norm. Sup. Pisa Cl. Sci. (4), 28 (1)(1999), 141-152.

9. Kowalski, Z.S., Invariant measures for piecewise monotonic transformation has a positive lower bound on its support, Bull de L'Academie Polonaise des Sci, Series des sciences mathermaticques, XXVII, No. 1, 1979, 53-57

10. Liverani, C., Decay of correlations for piecewise expanding maps, Jour. Statistical Physics, 78, Nos. 3/4, 1995, 1111-1129.

11. Liverani, C., Decay of correlations, Ann. of Math. (2) 142 (1995), No. 2, 239301.

12. Góra, P., Li, Zh., and Boyarsky, A., Harmonic average of slopes and the stability of acim, preprint.

13. Li, Zh., W-like maps with various instabilities of acim's, available at http://arxiv.org/abs/1109.5199

14. Li, Zh., Góra, P., Boyarsky, A., Proppe, H. and Eslami, P., A Family of Piecewise Expanding Maps having Singular Measure as a limit of ACIM's, accepted to Ergodic Th. and Dyn. Syst.

15. Schmitt, B., Contributions a l'étude de systemes dynamiques unidimensionnels en théorie ergodique, Ph.D. Thesis, University of Bourgogne, 1986. 